\title{
Q-Initiativen - eine Bestandesaufnahme der medizinischen Qualitätsarbeit in der Schweiz
}

Seit 2007 dokumentiert die FMH Qualitätsaktivitäten auf ihrer Website (www.fmh.ch $\rightarrow$ Qualität $\rightarrow$ Qualitätsinitiativen). Die Verbindung der Schweizer Ärztinnen und Ärzte hat damit eine Plattform für Wissensvermittlung und Vernetzung von Qualitätsbestrebungen geschaffen. Diese Plattform zeigt die Bandbreite der medizinischen Qualitätssicherung und -entwicklung in der Schweiz auf. Ausgewählte Qualitätsinitiativen, die beispielsweise von hohem öffentlichem Interesse sind oder eine Lücke füllen, werden zudem in Form eines Kurzporträts in der Schweizerischen Ärztezeitung publiziert.

\section{mediX - Guidelines und Gesundheitsdossiers}

Felix Huber

Korrespondenz; mediX schweiz Dr. med. Felix Huber Rotbuchstrasse 46 CH-8037 Zürich Tel. 0443665375 Fax 0443665069

\author{
Das Wichtigste in Kürze \\ - mediX hat über 70 Guidelines und 13 Gesund- \\ heitsdossiers entwickelt. \\ - Diese dienen der Optimierung der Patienten- \\ betreuung in der hausärztlichen Praxis. \\ - Sie werden laufend erweitert und aktuali- \\ siert. \\ - Der Wissenszuwachs wird mittels Fallbeispie- \\ len überprüft. \\ - Alle Guidelines und Gesundheitsdossiers \\ stehen online zur Verfügung. \\ - Die Entwicklung der Guidelines und Gesund- \\ heitsdossiers wird von Ärztenetzwerken finan- \\ ziert.
}

\section{Beschreibung}

mediX Schweiz hat über 70 Guidelines und 13 Gesundheitsdossiers entwickelt. Die Guidelines und Gesundheitsdossiers werden von Experten zusammen mit einem professionellen Redaktionsteam erarbeitet und in den Qualitätszirkeln von mediX (380 Ärzte) diskutiert. Sämtliche Guidelines und Gesundheitsdossiers basieren auf einer systematischen Literaturrecherche und berücksichtigen die aktuellen Publikationen aus peer reviewed Journals. Der Inhalt der Guidelines wird alle zwei Jahre auf die aktuelle Evidenz geprüft und spätestens nach fünf Jahren überarbeitet. Der Wissenszuwachs, der durch die Guidelines entsteht, wird mit Hilfe von Fallbeispielen und Analysen des Verordnungsverhaltens geprüft. Die Guidelines stehen der Öffentlichkeit online zur Verfügung.

Ärzte nutzen die mediX Guidelines als Entscheidungshilfen für ihre tägliche Arbeit. Die Guidelines verdanken ihre Attraktivität dem Praxisbezug, der Kürze und der pointierten Stellungnahme («mediX empfiehlt»). Sie unterscheiden sich von spezialärztlichen Guidelines der Fachgesellschaften durch ihre Praxisrelevanz für die Grundversorgung. Zudem sind die Guidelines frei von Sponsoring und Interessenkonflikten mit der Industrie. Die Kosten für die Erarbeitung der Guidelines werden über die Einnahmen aus den Budgetmitverantwortungsverträgen von den Ärztenetzen selber bezahlt.

\section{Eignung \\ Grundversorger, Hausärzte, Patienten}

\section{Finanzierung}

Das Budget von 150000 Franken jährlich wird von den fünf beteiligten Ärztenetzwerken finanziert.

\section{Weitere Informationen}

www.medix.ch 